This item was submitted to Loughborough's Research Repository by the author.

Items in Figshare are protected by copyright, with all rights reserved, unless otherwise indicated.

\title{
Computational optimisation of the thermal fusion bonding process in porous fibrous media for improved product capacity and energy efficiency
}

\section{PLEASE CITE THE PUBLISHED VERSION}

http://dx.doi.org/10.1177/0954408912457611

\section{PUBLISHER}

(C) IMechE. Published by Sage.

\section{VERSION}

AM (Accepted Manuscript)

\section{PUBLISHER STATEMENT}

This work is made available according to the conditions of the Creative Commons Attribution-NonCommercialNoDerivatives 4.0 International (CC BY-NC-ND 4.0) licence. Full details of this licence are available at: https://creativecommons.org/licenses/by-nc-nd/4.0/

\section{LICENCE}

CC BY-NC-ND 4.0

\section{REPOSITORY RECORD}

Peksen, Murat, Memis Acar, and W. Malalasekera. 2019. "Computational Optimisation of the Thermal Fusion Bonding Process in Porous Fibrous Media for Improved Product Capacity and Energy Efficiency". figshare. https://hdl.handle.net/2134/25688. 


\title{
Computational optimisation of the thermal fusion bonding process in porous fibrous media for improved product capacity and energy efficiency
}

\author{
M Peksen, M Acar and W Malalasekera
}

\begin{abstract}
A comprehensive parametric study to aid in optimising the thermal fusion bonding process in porous fibrous media is introduced. A systematic approach considering computational fluid dynamics, design of experiments and statistical process control is attempted. The effects of several operating and design parameters on the thermal bonding performance have been systematically investigated. An experimentally validated two-dimensional computational fluid dynamics model based on the theory of porous media has been used for the study. The air velocity and the conveyer belt porosity are determined to be showing the greatest optimisation potential for higher production rates. The optimum thermal fusion bonding process for the highest production rates and energy efficiency has been determined considering the thermal bonding time and percentage increase in total web energy absorption. Another outcome is the improved product quality associated with the improved temperature gradients within the fibrous web.
\end{abstract}

Keywords: nonwoven, thermal bonding, porous media, computational fluid dynamics, statistical process control, design of experiments

\section{Introduction}

Nonwovens are a sheet, web, or batt of natural or manmade fibres or filaments, excluding paper, that havenot been converted into yarns, and that are bonded to each other by any of several means ${ }^{1}$, including mechanical, chemical, thermal; especially, fusion through convective hot air, as it is considered for this study. Details of an industrial thermal bonding machine and the bonding process are given by Peksen et $\mathrm{al}^{2,3}$. A schematic of a typical thermal bonding machine is illustrated in Figure 1.

The machine comprises a rigid perforated rotating drum, a wire-mesh drum cover and a wire-mesh looped conveyor belt. The drum and drum cover are enclosed by a hood with an open section to enable the conveyor belt and the web to enter and exit the machine. The porous fibrous web enters the machine together with the conveyor belt, from room temperatures, and is then subjected to convective hot air flow, which is at or just above the melting temperature of the sheath fibre material. The web is located such that it is wrapped around the drum, sandwiched between the conveyor belt and the drum cover, first travelling through the machine's heating zone facilitating the bonding of the contacting fibres, and then it is briefly subjected to a cool air flow before leaving the machine. The convective hot air first flows through conveyer belt, then through the nonwoven web (porous) and finally through the drum cover and the perforated drum. All these elements enter the heating zone at different but much lower temperatures than the air temperature, thus absorbing significant amount of energy from the air. ${ }^{3}$ 


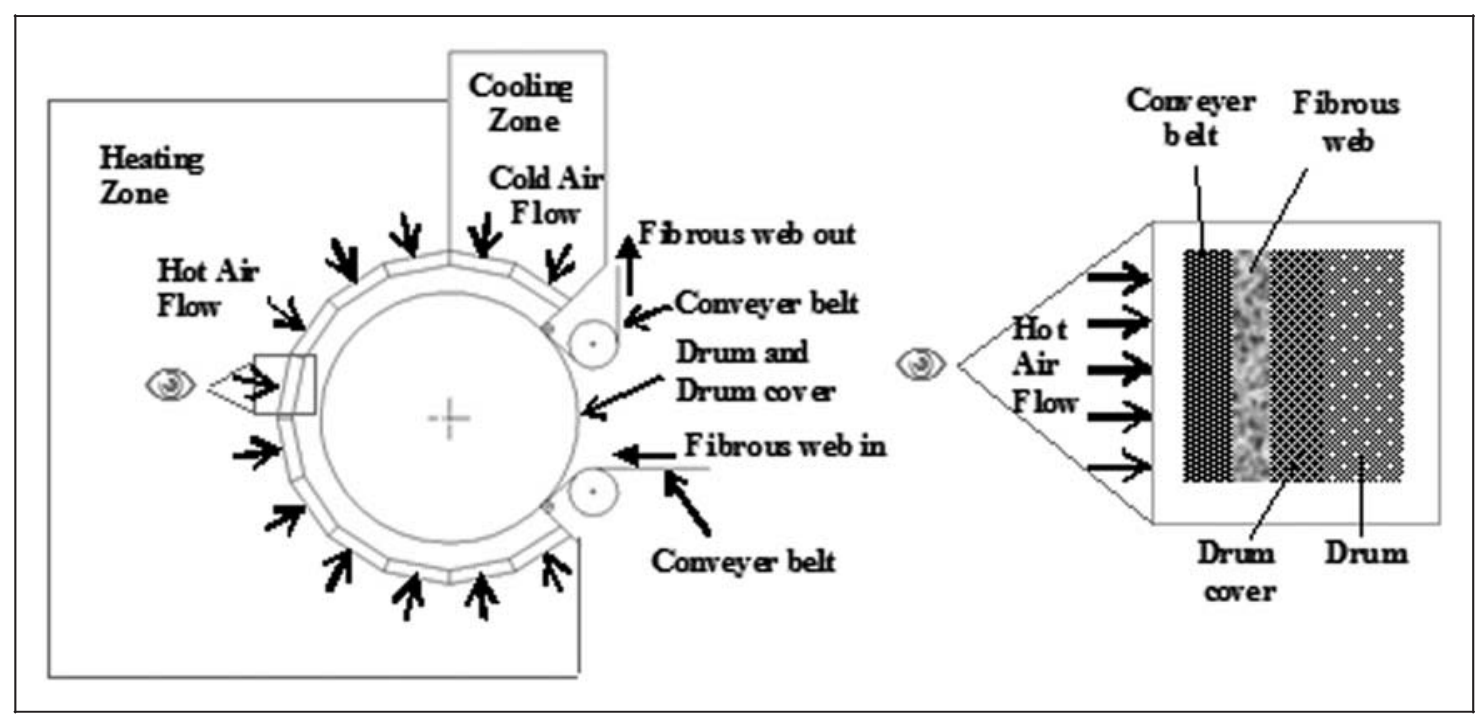

Figure 1. Schematic view of a typical thermal bonding machine and components ${ }^{3}$.

During the past years, the increasing demand on higher production rates and energy efficiency has motivated interest in the development of reliable design strategies that aim to optimise the through hot air bonded nonwoven process. This requires the right adjustment of the machine settings and the access to reliable and valid optimisation data. The optimisation and level of improvement is dependent upon a thorough under- standing of the thermofluid flow behaviour and the related parameters affecting the process. To attempt an optimisation, assessing experimentally the process parameters is prohibitive. On the other hand, the use of computational modelling for process synthesis and optimisation has shown significant benefit. The optimisation goal should consist of a cost-efficient weighted combination of both approaches. However, a successful industrial optimisation requires more, i.e. a strategic approach linking the performed measurements and computational techniques. Design of experiments (DOE) has been an invaluable tool to peruse process parameters systematically, thus reducing the assessment time and number of investigations needed. A strategic trio approach combining measurements, DOE and computational techniques has been introduced by Peksen et al. ${ }^{4}$ to optimise complex industrial processes. To employ such process models, it is essential to employ an experimentally validated model, in order to successfully carry out the optimisation, and to evaluate whether output from the process models can be used or not.

The comprehensive and flexible optimisation opportunities provided by the computational fluid dynamics (CFD) technique, comprising micro-, meso- and macro-modelling in addition to experimental validation of the thermal bonding process was elucidated in our previous studies. ${ }^{2,3,5,6}$ It was shown to be an excellent tool for ensuring reliable and valid data early in the process, hence reducing the need for additional experimental data. In this study, a comprehensive parametric study has been introduced to aid in the optimisation of the thermal fusion bonding process in porous fibrous media. In particular, attention is given to improve the production rate and energy efficiency.

\section{Methodology}

To systematically study the effects of several operating and design parameters on the thermal bonding performance, a combination of DOE, CFD and statistical process control (SPC) is employed. The desired conditions are to obtain higher nonwoven (fibrous web) production rates together with optimum product properties. The previously proposed experimentally validated two-dimensional (2D) CFD model $^{3}$ based on the continuum theory of porous media is used. For details about the governing equations and the derivation of the flow properties together with the effective material properties, computational model details, it is referred to this reference.

The computational model as to give a brief review, considers all thermal fusion bonding machine components, including the conveyer belt, porous web, drum cover and the drum. All components are stacked on top of each other. The transient problem considers a quasi- transient approach in which a $5^{\circ}$ radial sector of the geometry has been used. As a section is used, periodic boundary conditions are utilised. The model is subjected to thermo-fluid flow for a period of time that 
corresponds to the dwell time of $236^{\circ}$ segment of the machine in the heating zone, based on the speed of the drum. A structured computational mesh of 8241 nodes is employed. The commercial code FLUENT ${ }^{\circledR}$ has been used for the analyses.

The analyses were carried out by selecting one basic configuration varying each governing parameter within the range of the physical situations of interest. The basic configuration simulates the validated model for a $200 \mathrm{~g} / \mathrm{m}^{2}$ nonwoven fabric with an experimentally determined air inlet velocity of $0.665 \mathrm{~m} / \mathrm{s}$ and an air temperature of $225^{\circ} \mathrm{C}$. The web was assumed to be made of sheath-core type bi-component fibres of nylon (PA6; polyamide) sheath and polyester (PET) core with a nonwoven thickness of $0.909 \mathrm{~mm}$ corresponding to $200 \mathrm{~g} / \mathrm{m}^{2}$. The melting point of the nylon sheath was experimentally determined as 221 ${ }^{\circ} \mathrm{C}$. The conveyor belt speed, in other words the web throughput speed was $5 \mathrm{~m} / \mathrm{min}$. Porosities for the nonwoven, belt and the drum cover are considered to be as $0.88,0.43$ and 0.70 , respectively. Results obtained from these parametric studies will enable to identify the critical parameters for thermal bonding performance, hence being a reference for optimising the machine settings. Moreover, the optimum process for the highest production rates and energy efficiency is determined.

\section{Numerical parametric study using DOE}

To investigate the effect of major parameters on the bonding time an experimental design plan (DOE) based on numerical results is set up. The numerical parametric study performed in this section, the bonding time of the porous nonwoven fabric is to be studied with respect to its dependence on five factors each set at two levels:

(A) air inlet velocity

(B) air temperature

(C) fabric porosity

(D) conveyor belt porosity

(E) drum cover porosity

In this context, a factor is a numerical variable, and a result is the quantitative prediction of the parameter of interest. The bonding time is considered to be the time the PA6 sheath of the bi-component fibre reaches its melting temperature, i.e. $221{ }^{\circ} \mathrm{C}$. The porous nonwoven fabric is the product of the thermal bonding process; therefore, special attention is given to the effect on the temperature distribution, bonding time and the temperature gradients within the fabric. To investigate the parameters affecting these properties, an assembly of fractional factorial analyses with a half-fraction of a $2^{5}$ design with the defining relation $I=A B C D E$ is implemented.

This design is chosen as to be appropriate because it has a high resolution consistent with the degree of fractionation required. For half-fraction designs, this generally corresponds to choosing the defining contrast as the highest order interaction. The high resolution reduces the influence of aliasing on effect estimation and interpretation, particularly in respect of main effects and lower order interactions. It also provides for less restrictive assumptions in respect of which inter- actions require to be assumed negligible in the assessment of the associated effect estimates. To avoid referring to contrast expressions each time, a basic design approach can be used for treatment combination assignment in fractional factorial designs (FFDs).

The method is based on using a full $2^{k-p}$ design in the first $k-p$ factors and adding to this design the columns for the additional factors from the combination of the plus and minus signs associated with each design generator, for each row of the basic design. The design is to be a $2^{5-1}$ FFD with $k=5$ with defining relation $I=\mathrm{ABCDE}$. The simulations required to examine the process is sixteen 16 with the -1 exponent signifying that the design will be a half-fraction $(p=1)$. The assignment of the treatment combinations to the fractions can be carried out using the basic design approach. This entails using the full $2^{5-1}=2^{4 \text { design }}$ in the first four factors $A, B, C$ and $\mathrm{D}$, which allows for 16 runs. The design has been written in standard order for convenience though, normally, design implementation requires the treatment combination order to be randomised.

To find the levels of the fifth factor $\mathrm{E}$, the defining relation $I=\mathrm{ABCD}$ for the additional factor $E$ is solved, i.e. $E=A B C D$, which is referred to as the design generator. The levels of $\mathrm{E}$ for each treatment combination will correspond to the product of the plus and minus signs of the A, B, C and D columns (Table 1). The outcome to this is displayed in column $\mathrm{E}$, where the response is the bonding time of the nonwoven fabric.

This set up offers the promise of the shortest time in producing and testing the process rapidly. The values of low and high level for each parameter used are presented in Table 2.

The choice of the high and low values of the air velocity are determined such that $0.665 \mathrm{~m} / \mathrm{s}$ is chosen as the lower value which is the average air velocity measured on the machine, and $2 \mathrm{~m} / \mathrm{s}$ for the high value. The temperature values and the porosity ranges are feasible values suggested by the industrial partner Colbond bv. 
Table 1. Fraction and simulation data from $2^{5-1}$ two-level FFD with defining contrast $I=A B C D E$.

\begin{tabular}{llllllllllll}
\hline Run & A & B & C & $D$ & $E=A B C D$ & Run & A & $B$ & $C$ & $D$ & $E=A B C D$ \\
\hline I & - & - & - & - & + & 1 & 0.665 & 225 & 0.80 & 0.30 & 0.80 \\
2 & + & & - & - & - & 2 & 2 & 225 & 0.80 & 0.30 & 0.40 \\
3 & - & + & - & - & - & 3 & 0.665 & 235 & 0.80 & 0.30 & 0.40 \\
4 & + & + & - & - & + & 4 & 2 & 235 & 0.80 & 0.30 & 0.80 \\
5 & - & - & + & - & - & 5 & 0.665 & 225 & 0.92 & 0.30 & 0.40 \\
6 & + & - & + & - & + & 6 & 2 & 225 & 0.92 & 0.30 & 0.80 \\
7 & - & + & + & - & + & 7 & 0.665 & 235 & 0.92 & 0.30 & 0.80 \\
8 & + & + & + & - & - & 8 & 2 & 235 & 0.92 & 0.30 & 0.40 \\
9 & - & - & - & + & - & 9 & 0.665 & 225 & 0.80 & 0.80 & 0.40 \\
10 & + & - & - & + & + & 10 & 2 & 225 & 0.80 & 0.80 & 0.80 \\
11 & - & + & - & + & + & 11 & 0.665 & 235 & 0.80 & 0.80 & 0.80 \\
12 & + & + & - & + & - & 12 & 2 & 235 & 0.80 & 0.80 & 0.40 \\
13 & - & - & + & + & + & 13 & 0.665 & 225 & 0.92 & 0.80 & 0.80 \\
14 & + & - & + & + & - & 14 & 2 & 225 & 0.92 & 0.80 & 0.40 \\
15 & - & + & + & + & - & 15 & 0.665 & 235 & 0.92 & 0.80 & 0.40 \\
16 & + & + & + & + & + & 16 & 2 & 235 & 0.92 & 0.80 & 0.80 \\
\hline
\end{tabular}

FFD: fractional factorial design.

Table 2. Low and high values of each parameter used for CFD analyses.

\begin{tabular}{lll}
\hline Parameter & Low $(-)$ & High \\
$\underline{(+)}$ & & \\
Air velocity $(\mathrm{m} / \mathrm{s})$ & 0.665 & 2 \\
Air temperature $\left({ }^{\circ} \mathrm{C}\right)$ & 225 & 235 \\
Fabric porosity & 0.80 & 0.92 \\
Conveyor belt porosity & 0.30 & 0.80 \\
Drum cover porosity & 0.40 & 0.80 \\
\hline
\end{tabular}

CFD: computational fluid dynamics.

\section{Results and discussion}

\section{Parameters influencing the respond variables}

The $2^{5-1}$ design for the bonding time study is an illustration of a resolution $\mathrm{V}$ design as the contrast within the defining relation $I=\mathrm{ABCDE}$ is of length 5 , i.e. a $2^{5-1}$ FFD. Designs of this type have no main effect aliased with any other main effect, two-factor inter-action, or three-factor interactions. This type of design allows for unique estimation of all main effects and two-factor interactions, provided that all higher order interactions can be assumed negligible. ${ }^{7}$

The analysis procedures associated with FFDs were conducted as exploratory (effect estimates and effect plots). The CFD predictions are presented in Table 3. The effect analyses are performed using directly SPC.

\section{Exploratory data analysis}

Exploratory data analysis was considered to gain an initial insight into the effect the factors have on the response. The run order plot suggested acceptably random responses. A definite reduction in bonding time was obvious with higher values of air velocity and conveyer belt porosity occurring at its high level. For a thorough investigation, an effect estimate analysis is performed.

\section{Effect estimate analysis}

When carrying out an effect estimation and assessment, the associated alias structure must be taken into account. Using appropriate contrasts for the FFD used, it was possible to generate the effect estimates for the main effects, and two-factor interactions. As to evaluate the effects of the parameters, it is referred to the SPC tool Pareto chart. The effect estimates being provided in the results were specified as

Effect estimate $=($ Effect contrast $) / 2^{k-p-1}$ where $p$ defines the level of fractionation.

Figure 2 displays the Pareto chart where the values in the $y$-axis are drawn as bars in descending order. Each bar is labelled with the associated value in the effect names.

The graphic represents the parameters affecting the bonding time. According to the results demonstrated, the important effects are clearly seen. It should be noticed that the Pareto chart does not consider the 
Table 3. Numerical parametric study results for the bonding time.

\begin{tabular}{|c|c|c|c|c|c|c|c|c|c|c|c|c|c|c|c|c|}
\hline CFD run & I & 2 & 3 & 4 & 5 & 6 & 7 & 8 & 9 & 10 & II & 12 & 13 & 14 & 15 & 16 \\
\hline Bonding time (s) & 26.4 & 7.2 & 18.1 & 4.8 & 25.9 & 6.8 & 15.8 & 4.9 & 19 & 2.1 & 5.7 & 1.5 & 10.3 & 2.2 & 5.2 & $\mathrm{I} .4$ \\
\hline
\end{tabular}

CFD: computational fluid dynamics.

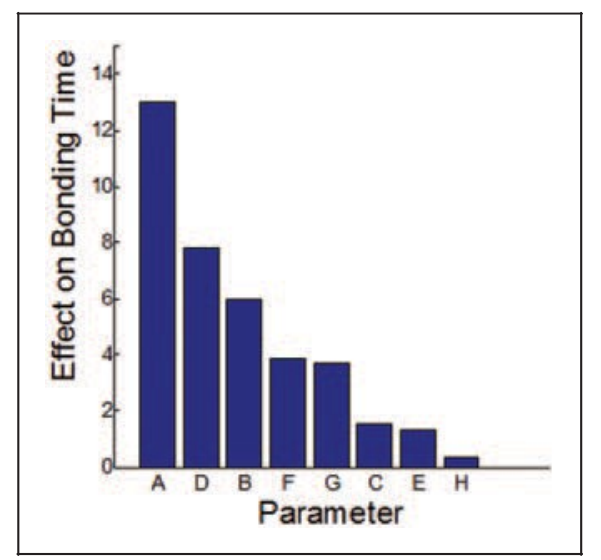

Figure 2. Numerical parametric study for defining parameters effecting the bonding time.

minus signs; therefore, in the analysis of the bonding time, the value of the effect refers to decreased bonding time.

The parameter labels F, G and $\mathrm{H}$ refer to the twofactor interactions of $\mathrm{AB}, \mathrm{AD}$ and $\mathrm{BD}$, respectively. Thus, it is clear that $A, D, B, F$ and $G$ have a great effect on the bonding time, whereas $\mathrm{C}, \mathrm{E}$ and $\mathrm{H}$, i.e. the porosities of the nonwoven web, the drum cover and the interaction of air temperature and belt porosity have less influence.

The interaction effects $F$ and $G$ are aliased. These can be shown by looking at the effect estimates of the contributing main effects presented. Considering the interaction $A B(F)$, effect $B$ is low signifying an important effect and effect $A$ appearing as more important, so it could be argued that the associated interaction is due to the effect of $\mathrm{A}$, and just underpinning its associated effect estimate. Another approach can be stated that in effect $A D(G)$, both main effects appear important so it is highly likely that the associated interaction will also be important. Hence, the numerical parametric study results for higher production rates, i.e. reduced bonding time suggests the use of increased air velocity or as an alternative, a conveyor belt with higher porosity rates. For an optimisation of the production rate combined with the energy efficiency, the total energy per unit mass of air considering both the kinetic and internal energy has to be considered besides the production rate; therefore, a desired optimisation comprises the consideration of both aspects.
Table 4. Detailed data set for bonding time and percentage increase in total energy.

\begin{tabular}{cccc}
\hline $\begin{array}{c}\text { CFD } \\
\text { run }\end{array}$ & $\begin{array}{c}\text { Bonding } \\
\text { time }(\mathrm{s})\end{array}$ & $\begin{array}{l}\text { Total } \\
\text { energy }()\end{array}$ & $\begin{array}{l}\text { Increase in total } \\
\text { web energy } \\
\text { absorption }(\%)\end{array}$ \\
\hline 1 & 22.1 & 12,012 & 0.00 \\
2 & 26.4 & 11,654 & -2.98 \\
3 & 7.2 & 12,539 & 4.38 \\
4 & 18.1 & 12,753 & 6.10 \\
5 & 4.8 & 14,027 & 16.77 \\
6 & 25.9 & 11,710 & -2.50 \\
7 & 6.8 & 12,542 & 4.40 \\
8 & 15.8 & 13,237 & 10.19 \\
9 & 4.9 & 14,025 & 16.75 \\
10 & 19 & 12,121 & 0.90 \\
11 & 2.1 & 12,542 & 4.40 \\
12 & 5.7 & 13,708 & 14.11 \\
13 & 1.5 & 14,027 & 16.77 \\
14 & 10.3 & 12,382 & 3.08 \\
15 & 2.2 & 12,541 & 4.40 \\
16 & 5.2 & 13,717 & 14.19 \\
17 & 1.4 & 14,028 & 16.78 \\
\hline
\end{tabular}

CFD: computational fluid dynamics.

\section{Process optimisation}

The parametric study performed in the 'Results and discussion' section demonstrated the power of the CFD technique combined with DOE and SPC in performing rapid process optimisation numerically by avoiding the need for additional experimental data. In this section, the computational analyses are performed in the given DOE order as illustrated in Table 1. The aim is to propose the optimum configuration of the examined parameters to optimise the current throughair bonding process performed on a pilot machine (Figure 1). Table 4 illustrates the detailed predicted results for the bonding time and total energy absorbed by the porous fibrous nonwoven web. The data set for the total energy is the amount of energy per unit mass. The energy is due to the thermo-fluid flow of the air and includes the internal energy and the energy due to the kinetics of the flow. 


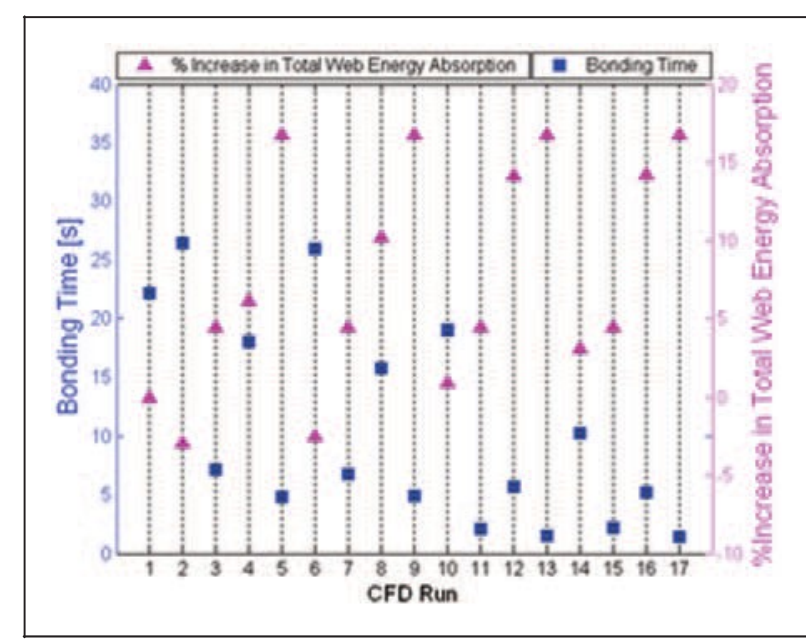

Figure 3. Optimum process for higher production rates and energy efficiency.

The percentage increase in total energy absorption of the web is the increase in energy of the CFD results 2-17 compared with the baseline configuration designated as CFD run number 1 . The baseline CFD analysis for the total energy is 12 012J, whereas the time for the area weighted average temperature of the web to reach $221^{\circ}$ is predicted to be $22.1 \mathrm{~s}$. The percent- age increase in total energy is considering this value as comparison. To determine optimum production rates and energy efficiency, a plot of two data sets and using both left- and rightside $y$-axes is created.

The results are illustrated in Figure 3. The bonding time of the nonwoven web is considered to be one parameter displayed on the left of the graphic, whereas the percentage increase in total web energy absorption (based on the base line simulation result) is plotted on the right scale of the $y$-axis.

The same range is used in the dependent variable, i.e. the number of the CFD analyses starting with the baseline prediction designated as run number 1 , and ending with the experimental design analysis results shown with run numbers $2-17$.

The results illustrate that the lowest bonding time is achieved for the configuration numbers 13 and 17 . However, when the energy absorptions are considered it is visible that due to the higher temperatures applied, the percentage of energy increase is not showing low values compared to the remaining configurations. The configurations 11 and 15 appear to be very good alternatives; especially, when the energy efficiency is considered. These configurations show a slightly higher bonding time (of approximately half a second), than the configurations 13 and 17, whereas the percentage increase in energy absorption is $12 \%$ less than the con-figurations 13 and 17. Comparing configurations 11 and 15 results in the same conclusion achieved.
The drum cover effect is negligible compared to the belt porosity, hence a configuration change of the drum cover is not needed under the performed conditions because the predicted results are very close to each other, thus configuration 11 with the higher value drum cover porosity can be chosen. Configurations 2 and 6 show the most efficient energy absorption rates compared to all configurations; however, those do not reach $221^{\circ} \mathrm{C}$ within the $22.4 \mathrm{~s}$ time interval, which is the time the web is subjected to heat in the pilot machine. Therefore, these are not considered to be useful configurations, in order to optimise the pilot machine. Figure 4 shows the comparison of the temperature distributions for the nonwoven depicting the current process conditions obtained using the basis line CFD model and the proposed optimised configurations.

Results show a significant improvement of the bonding time when the proposed optimised configurations are used. Higher temperature values are achieved faster as expected due to the higher air velocity rates. The air velocity has a direct effect on the heat transfer coefficient, hence increasing the heat transfer rates. Temperature distributions are very similar for the cases 11 and 15 due to reaching very fast steady state. Another important improvement achieved together with the optimised parameters is the expected optimised product quality, which is associated with the temperature distribution within the nonwoven structure (Figure 4(b)). The radial direction shows the temperature distribution starting from the belt side of the web to the drum cover side. It is clearly seen that the temperature gradient within the nonwoven is eliminated; hence, a uniform temperature distribution is enabled. Figure (a) illustrates the total energy absorbed by the nonwoven web.

The energy distributions show clearly the same trend as the temperature distribution. The prediction of the energy involves the rate of enthalpy and the kinetic energy leaving or entering the analysed system. Therefore, it is related to the specific enthalpy, which is a function of the temperature. This is why those graphics have the similar behaviour. Figure 5(b) shows the velocity distribution of the air within the web. The nonlinear behaviour of the velocity profile is due to the density variations. The density of the air is a function of the process temperature and the mixture content of the air.

As the air is treated as a mixture of dry air and water vapour, the density is changing during the heating process. It is decreasing as the temperature increases, thus causing also a change in pressure yielding in a nonlinear behaviour of the velocity. The differences of the velocity distributions between case 11 and case 15 are due to the porosity differences. Both cases 11 and 15 have the same belt porosity, thus the air 


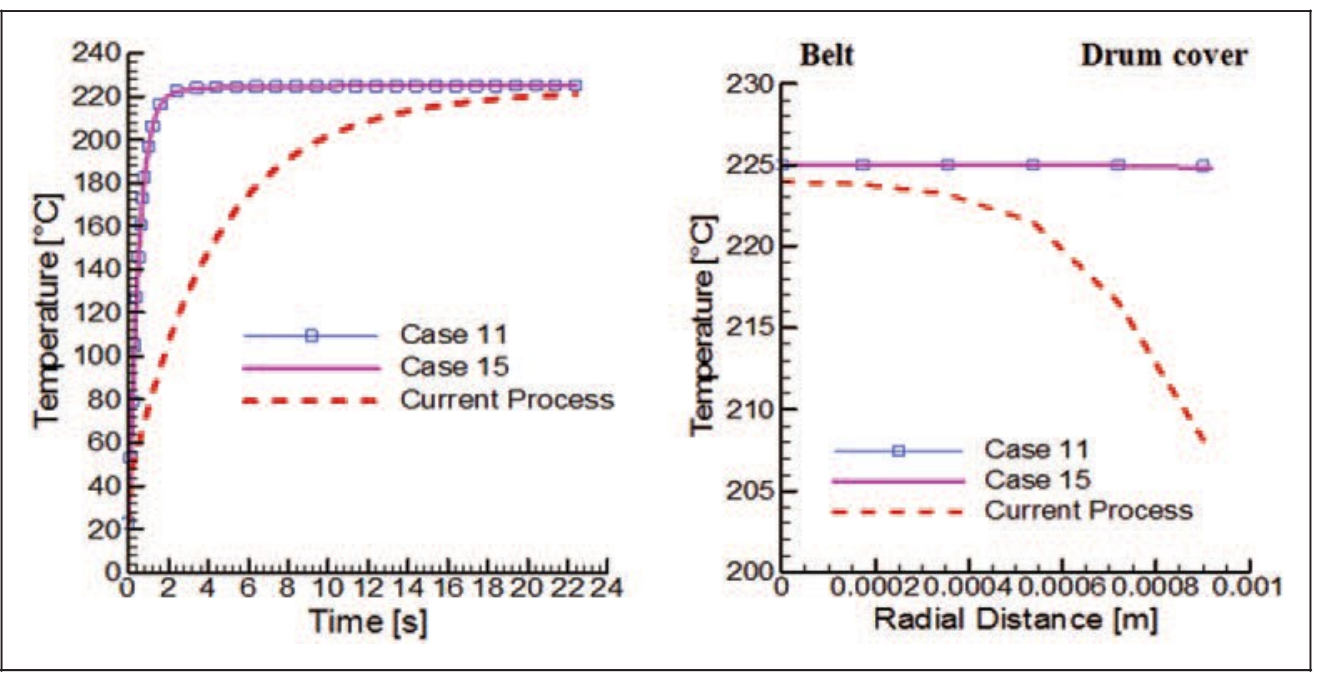

Figure 4. Comparison of current process and optimised process conditions: (a) fabric temperature distribution over time; (b) temperature distribution within the fabric.

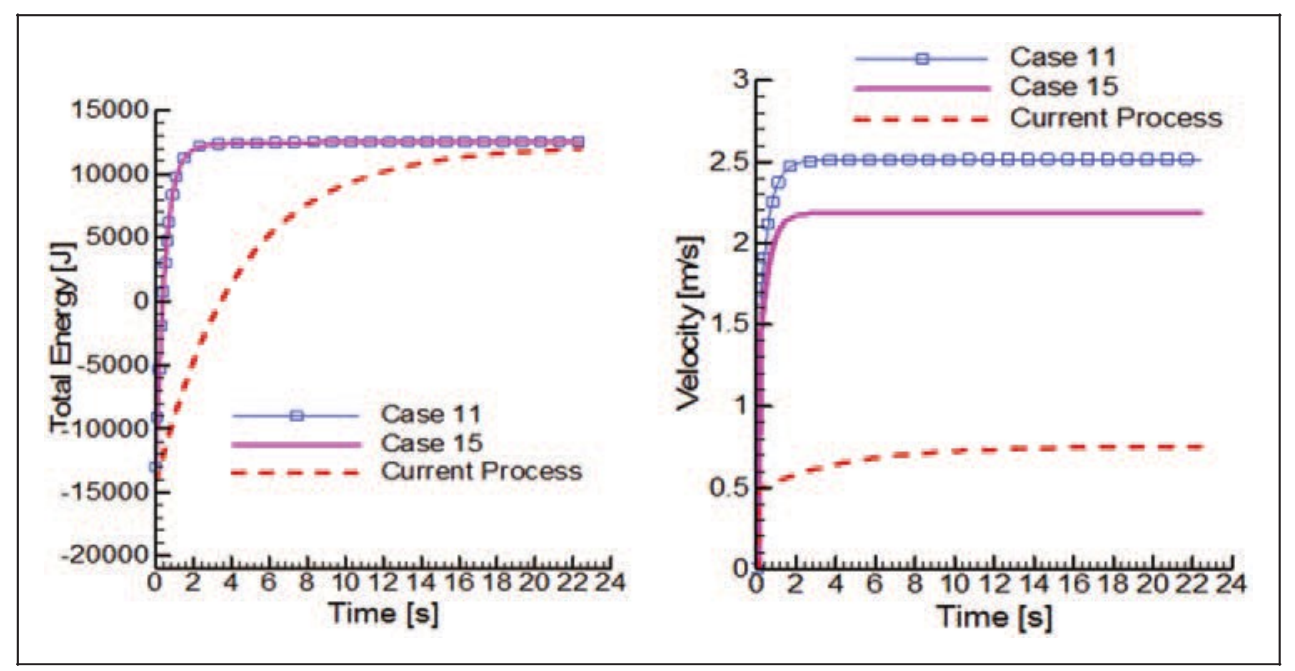

Figure 5. Comparison of current process and optimised process conditions: (a) total energy absorption; (b) velocity distribution.

velocity flowing through and out of the belt is the same and showing a value of $2.5 \mathrm{~m} / \mathrm{s}$. As the air enters the fabric in case 11, it flows through an environment having the same porosity, i.e. 0.80 , thus retaining the same velocity value. However, in case 15, the airflow enters a domain with an increased porosity of 0.92 . According to the conservation of mass, the increased flow area yields to a decrease in air velocity because the mass flow rate is constant.

\section{Conclusions}

In this article, the optimisation opportunities provided by the CFD technique coupled with DOE and SPC were used to perform a comprehensive parametric study to aid in the optimisation of the thermal bonding process performed on the pilot machine at COLBOND bv, the Netherlands. An experimentally validated 2D continuum model based on the porous media concept was used to study the effects of several operating and design parameters on the thermal bonding performance. The bonding time of the nonwoven fabric and the web energy absorption rate were studied with respect to their dependence on five factors each set at two levels: air inlet velocity, air temperature, fabric porosity, conveyor belt porosity and drum cover porosity. Results revealed that the air velocity has the greatest effect on the bonding time; thus, for higher production rates, the increase in air inlet velocity would be of advantage. This indicates a higher conveyor belt speed capability. 
As an alternative, the use of a conveyor belt with higher porosity rates would lead to higher production rates.

This could be achieved with just a slight increase in the total energy absorption by the nonwoven. Thermal gradients within the nonwoven could be improved, which would lead to a better product design. Another important outcome of the study was the negligible influence of the drum cover porosity on the bonding time. The effect analysis supports this issue by presenting the drum cover porosity as not being a parameter significantly effecting the bonding time. The predicted results showed that the belt porosity has a significant effect on the bonding time compared to the drum cover porosity. But as seen from the results of the detailed data of the parametric study and also of the effect analysis, the air inlet velocity has the highest impact on the bonding time.

\section{Funding}

This work received financial and technical support by COLBOND bv, The Netherlands.

\section{References}

1. Butler I. Nonwoven fabrics handbook. Cary, NC: INDA, 1999.

2. Peksen M, Acar M and Malalasekera W. Computational modelling and experimental verification of through-air bonding nonwovens process. In: Proceedings of the international nonwovens technical conference, Atlanta, GA, 24-27 September 2007, p.8.

3. Peksen M, Acar M and Malalasekera W. Computational modelling and experimental validation of the thermal fusion bonding process in porous fibrous media. Proc IMechE Part E: J Process Mech Eng 2010; 225(E3): 173-182.

4. Peksen M, Peters Ro, Blum L, et al. Design and optimisation of SOFC system components using a trio approach: Measurements, design of experiments, and 3D computational fluid dynamics. J Electrochem Soc, SOFC-XI 2009; 25(2): 1195-1200.

5. Hossain M, Acar M and Malalasekera W. A mathematical model for transverse airflow and heat transfer through fibrous webs. Proc IMechE Part E: J Process Mech Eng 2005; 219: 357-366.

6. Hossain M, Acar M and Malalasekera W. Modelling of the through-air bonding process. J Eng Fibers Fabr 2009; 4(2): $1-8$.

7. Gardiner PW and Gettinby G. Experimental design techniques in statistical practice. Chapter 7. West Sussex, England: Horwood Publishing, 1998.

\section{Appendix}

Notation

I defining relation of the fractional design

$k$ factor

$p \quad$ number of generator 